\title{
Organization and Use of the Mental Lexicon by Deaf and Hearing Individuals
}

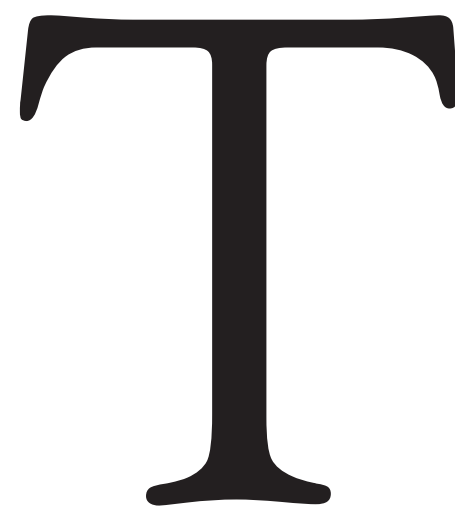

Marc Marschark, Carol Convertino, Cathy McEvoy, and Allison Masteller

MARSCHARK IS A PROFESSOR IN THE Department of Research, National TECHNICAL INSTITUTE FOR THE DEAF (NTID), ROCHESTER INSTITUTE OF TECHNOLOGY, RoChESTER, NY, AND IN THE DEPARTMENT OF Psychology, UnIVERSITY OF ABERDEEN, Aberdeen, Scotland. Convertino is a RESEARCH ASSOCIATE IN THE DEPARTMENT OF RESEARCH, NTID. McEVOY IS A PROFESSOR in the Department of Gerontology, UnIVERSITY OF SOUTH FLORIDA, TAMPA. Masteller is a teacher at the ROChester SCHOOL FOR THE DEAF, ROCHESTER, NY.

WO EXPERIMENTS EXPLORED the taxonomic organization of mental lexicons in deaf and hearing college students. Experiment 1 used a single-word association task to examine relations between categories and their members. Results indicated that both groups' lexical knowledge is similar in terms of overall organization, with associations between category names and exemplars stronger for hearing students; only the deaf students showed asymmetrical exemplar-category relations. Experiment 2 used verbal analogies to explore the application of taxonomic knowledge in an academically relevant task. Significant differences between deaf and hearing students were obtained for six types of analogies, although deaf students who were better readers displayed response patterns more like hearing students'. Hearing students' responses reflected their lexical organization; deaf students' did not. These findings implicate the interaction of word knowledge, world knowledge, and literacy skills, emphasizing the need to adapt instructional methods to student knowledge in educational contexts.

How is human knowledge organized? This question has been asked by philosophers, educators, linguists, and psychologists from a variety of theoretical and pragmatic perspectives. Perhaps the most consistent finding is that knowledge involves hierarchical, taxonomic categories. Human knowledge about living beings, for example, is organized into categories of animals and plants, and further into categories of fish and birds, pets and farm animals, trees and flowers, and so on. Taxonomic organization has been observed through its impact on recall at least since Bousfield (1953) and has been measured through various association tests.

Deese (1966), for example, showed that when students gave single-word associations to words, the most frequent responses included category superordinates (e.g., animal to the word dog), subordinates (e.g., dog to animal), and coordinates (e.g., cat to dog). Deese pointed out that these responses reflect both formal, logical learning of groupings with agreed-upon titles (such as animal) and informal categories (such as "things one can carry"). This is logical, perhaps, but later studies showed that the organization of knowledge is affected by an individual's personal and cultural experience. For example, a comparison of aboriginal and white Australian adolescents (Drinkwater, 1972), and another of French-speaking and Englishspeaking Canadians (Vikis-Freibergs \& Freibergs, 1976), both indicated greater differences than are observed within a single-language cultural group, although there still was considerable agreement across the two groups in both cases. Yoon et al. (2002) similarly found variation in the responses given 
in China and the United States, particularly for categories such as food items that could vary on a regional basis.

Across cultures, and even among groups within a culture, the experiences individuals have with different categories and their labels may vary extensively, and formal (i.e., school) training in category membership may vary as well. This is of particular interest in regard to deaf students, for a variety of reasons. More than $95 \%$ of deaf children have hearing parents (Mitchell \& Karchmer, 2004), and the concomitant language and experiential differences between these children and deaf children with deaf parents is well documented (e.g., Marschark, 1997; Moores, 2001). Particularly in light of the wide range of educational content, quality, and communication experienced by deaf students, it therefore would not be surprising to find that these students' knowledge and its organization might vary from that of hearing students. Recognition of such variation would simply be an acknowledgment of differences in deaf students' experiential and educational backgrounds, while the onus would be on investigators and educators to develop methods of teaching to those differences, rather than ignore them (Marschark \& Lukomski, 2001). However, such findings are not without theoretical complexity.

Kerr and Johnson (1991) compared the associative responses obtained from small groups of blind and sighted college students, and found moderate similarity. Interestingly, the degree of commonality of responses did not seem to vary on the basis of whether the stimulus was a concept that is normally experienced visually, such as sky or green, or one that is also experienced with the other senses, such as cigar or engine. Similar findings were obtained by McEvoy, Marschark, and Nelson
(1999). They obtained single-word associations from deaf college students and compared them to responses of hearing students by means of the University of South Florida (USF) Word Association Norms (Nelson, McEvoy, \& Schreiber, 1998). Although McEvoy et al. found an extensive overlap in responses from the two groups ( $r=$ .77), significant differences on all of the dimensions analyzed indicated that deaf students had more heterogeneous conceptual organization than their hearing peers and weaker associative relations among concepts. Nonetheless, no differences were obtained as a function of whether or not the words were sound related (e.g., banjo vs. closet).

\section{Application of Taxonomic Knowledge by Deaf Students}

A variety of studies have examined the link between the organization of conceptual knowledge and memory performance in hearing individuals. But relatively few have done so with deaf individuals, and none have examined the way in which differences observed in deaf students relative to hearing students might affect academic performance. Several studies conducted through the 1970s, for example, indicated that deaf and hearing children viewed familiar objects in similar ways, as reflected in how they sorted items into groups (e.g., Koh, Vernon, \& Bailey, 1971; Liben, 1979; Tweney, Hoemann, \& Andrews, 1975). Although all of these studies involved simple, highly familiar stimuli, deaf students in grade school through college tended not to use conceptual or taxonomic information in recall and typically remembered less than hearing peers, even when they did use such information (see also Strassman, Kretschmer, \& Bilsky, 1987).
More recently, Marschark and Everhart (1999) examined the use of taxonomic information in problem solving by deaf and hearing students. They used a form of the Twenty Questions game with deaf and hearing students from age 7 years to college age. Each participant saw a matrix of 42 pictures that included things in different taxonomic categories (e.g., animals), functional categories (e.g., tools), and perceptual categories (e.g., red things). Hearing students were more likely than deaf peers to solve the game at all ages, although the difference was not significant among college students. ${ }^{1}$ Hearing students also were significantly more likely to ask questions that involved taxonomic information (e.g., "Is it round?" or "Is it an animal?"), while deaf students did not apply any consistent strategies. As in the case of memory studies, these results reveal both the heterogeneity of deaf students' conceptual knowledge and the relatively lesser likelihood that they will apply the knowledge they do have (Liben, 1979; Strassman, 1997).

Because knowledge of categorical structure develops as a consequence of an individual's formal and informal experiences (Landauer \& Dumais, 1997; Nelson, 1999), findings like those obtained by Marschark and Everhart (1999) could result from either deaf students' weaker associations among concepts or their lesser likelihood of making use of (or being aware of) strategies appropriate to particular task situations. In either case, as in cross-cultural studies, one would expect experiential differences to be mirrored in subtle or not-so-subtle variations in situations that tap that underlying knowledge. Taxonomic knowledge is not the sole form of associative organization used in understanding the world, but it represents an important mode of thinking about 
and interacting with objects and concepts in daily life (Goswami, 1992). Those relations indicate implicit or explicit links between items, how one item might substitute for another, and where items fit into the human environment. They also play a central role in reading comprehension and learning, both in formal educational settings and in everyday functioning in the world.

Given previous findings that deaf students are less likely than hearing peers to use categorical knowledge in laboratory problem-solving tasks (even when they have that knowledge), it thus should not be surprising that deaf students often fail to apply it in functionally similar educational contexts (Strassman, 1997; Strassman et al., 1987). Indeed, the expectation that deaf students have the same knowledge as hearing peers and use it in similar ways is a faulty assumption underlying much of mainstream education (Marschark \& Lukomski, 2001). Alternatively, deaf and hearing individuals may be equally likely to apply taxonomic knowledge in both laboratory and educational settings, but that knowledge may be relatively less coherently organized by deaf students or less accessible to them, and therefore less likely to support comprehension and learning effectively (McEvoy et al., 1999). The two experiments in the present study examined these alternatives through a single-word association task, of the kind commonly used in laboratory studies of knowledge organization, and a verbal analogy task, as found on standardized achievement and academic admission examinations.

\section{Experiment 1: A Single-Word Association Task}

Marschark and Everhart (1999) found that deaf students were less likely than hearing peers to ask categorical ques- tions (e.g., "Is it an animal?") in playing Twenty Questions. They concluded that deaf students are less likely than hearing peers to activate superordinate information in such contexts. Alternatively, deaf students may not have any particular difficulty in automatically activating categorical information in response to exemplars, but may not spontaneously use that information to facilitate performance (Goswami, 1992; Liben, 1979; Ottem, 1980).

Experiment 1 was designed to examine deaf students' use of taxonomic information more closely. Single-word associations were given by deaf students in response to a selected set of category names (superordinates) and category members (subordinates). In a single-word association task, common responses given by large numbers of individuals are assumed to reflect both high levels of familiarity and strong associations with the stimulus, at least for the members of the tested group. Infrequently given responses are assumed to be more weakly associated with the stimulus, less familiar to the tested group, or both. Similarly, stimuli that produce strongly interconnected sets of responses are assumed to be familiar and to be representative of wellorganized knowledge, whereas stimuli that produce weak-agreement responses or that many individuals fail to respond to are assumed to be less familiar and to represent less organized concepts (Chaffin, 1997). By comparing the responses of different groups of individuals, investigators can measure knowledge differences that might underlie differential cognitive performance in such areas as memory, problem solving, reading, and writing.

In Experiment 1, single-word association responses given by the deaf students were compared with responses that had been given by hearing students. On the basis of earlier findings, deaf students were expected to be (a) more variable in their response sets than hearing students (McEvoy et al., 1999) and (b) less likely than hearing students to respond with category names to exemplars (Marschark \& Everhart, 1999).

\section{Method \\ Participants}

The participants in Experiment 1 were 131 deaf students from the various colleges of the Rochester Institute of Technology (RIT) who volunteered in response to flyers or personal contacts; all received compensation for participating. Data on hearing students were drawn from the USF Word Association Norms (Nelson et al., 1998), described in the opening section of the present article.

\section{Materials}

The stimuli were 40 common English words selected from the USF Word Association Norms (Nelson et al., 1998): 20 category names for which the primary associate in the hearing norms was an exemplar of that category (e.g., reptile - snake) and 20 exemplars for which the primary associate was a category name (e.g., banana fruit). Potential stimuli were examined by two individuals not connected with the present study who had many years' experience teaching English to deaf students. Words that the two experts agreed were unfamiliar to many deaf students were replaced. Several words so designated by only one of the experts were left in the list to provide a wider range of familiarity.

\section{Procedure}

Most testing occurred in group settings (i.e., classes and meetings of student organizations), so as to ensure as broad a representation as possible of the 
deaf student population. Explanation of the task, the answering of questions, and task administration were both signed and spoken. Selected stimuli were presented in two columns on a single page; the 20 category names and 20 exemplars were randomly intermixed. Students were asked to write the first word that came to mind in a blank space next to each word. When they had finished, they were asked to circle any words that were unfamiliar. The task was not timed, but participants were encouraged to work through it without spending too much time on any single word. It was emphasized that spelling was not important.

Because of the importance of taxonomic relations in reading and other academic tasks (Goswami, 1992), literacy-related information was obtained from the deaf students' records related to their performance on the American College Test (ACT, normally required for students entering RIT), the Scholastic Aptitude TestVerbal (SAT-V), the Michigan Test of English Language Proficiency, the California Reading Comprehension Test, and the National Technical Institute for the Deaf (NTID) Reading Test and NTID Writing Test (both used for course placement). Such information was not available on the hearing (University of South Florida) students.

\section{Results and Discussion}

For a student's data to be included in the analyses, valid responses were required to at least 20 of the 40 words. This criterion eliminated 5 of the 131 deaf students. Unless otherwise noted, all results reported for Experiment 1 were significant at the .05 level.

Although the activation of related associations is normally thought of as automatic (Nelson et al., 1998), individual words may be recognized and even evoke some associations without having sufficient strength to lead to a particular response (or to comprehension or recall in other tasks; Liben, 1979; Strassman et al., 1987). To provide a fair comparison with the hearing norms, in which essentially all students responded to all words, the strength of the primary associations for deaf students was calculated by dividing the frequencies of the common responses by the total number of valid responses. The number of valid responses for each stimulus word was obtained by subtracting from the total number of deaf participants (126) the number of words that were circled (i.e., were apparently unfamiliar to the test taker), the number of spaces that were left blank, and the number of responses that were discarded because they were illegible. For example, for the category name pasta, 32 deaf students responded with the hearing primary associate, spaghetti. One student circled the word and two left it blank, leaving 123 valid responses. Thus, the response strength of spaghetti was .26 $(32 \div 123)$.

One set of analyses compared primary associates in the hearing norms with those given by the deaf students. Overall, the response patterns were similar, as deaf and hearing students' primary associates matched for 35 of the 40 stimulus words. As predicted, however, the deaf students showed more variability in their responses than the hearing students: Of the primary

Table 1

Students' Responses, Single-Word Association Task, Experiment 1

\begin{tabular}{|lccccc|}
\hline & \multicolumn{2}{c}{ Category responses } & \multicolumn{2}{c|}{ Exemplar responses } \\
Associative strength (proportional) & $M$ & $S D$ & $M$ & $S D$ \\
\hline \multicolumn{1}{c}{ Hearing students } & .41 & .17 & .47 & .20 \\
\hline Deaf students & .30 & .15 & .38 & .16 \\
Deaf-hearing response matches & 37.35 & 18.64 & 48.10 & 19.59 \\
Designated "unfamiliar" & 4.70 & 9.69 & 7.60 & 10.84 \\
Left blank & 0.85 & 0.75 & 1.10 & 1.55 \\
Discarded & 0.30 & 0.66 & 0.40 & 0.60 \\
\hline
\end{tabular}

associates given by deaf students, there were only 10 for which their responses matched the primary associates of the hearing norms with at least a .50 (proportional) strength or higher. In the hearing norms, 14 stimuli elicited responses with a strength of .50 or greater: Seven of those stimuli were exemplars (a proportion equal to that elicited from the deaf students) and 7 were category names (more than twice as many, proportionately, as were elicited from the deaf students). Overall, the correlation between strengths of associations from deaf and hearing responses was significant, $r(38)=.64$ (see Table 1). At the same time, the deaf students' primary associations were weaker than those of the hearing students, $t(39)=3.32$. These findings thus replicate those of McEvoy et al. (1999) showing significantly greater heterogeneity in the concept knowledge of deaf students than in that of hearing students, despite the qualitative similarity of the two sets of knowledge. In particular, McEvoy et al. found an overlap of .77 in responses to the diverse word set used in their study, while the more restricted set used in Experiment 1 yielded an overlap of .87 .

Because of our interest in possible differences in the linkages between categories and their exemplars made by deaf and hearing students, separate analyses were conducted for exemplar and category stimuli. Contrary to our prediction, the strength of the associa-

\section{4}


tions between exemplars and category names did not differ for the deaf and hearing groups, $t(19)=1.68$, ns. Deaf students, in fact, were more likely to respond to an exemplar with a category name than they were to respond to a category name with an exemplar, $t(112)=4.67$. Their associative connections from category names to exemplars were not as strong as hearing students', $t(19)=3.03$, however, even though they followed the same pattern, $r(18)=.59$. Strengths of primary associates of category and exemplar stimuli did not differ for the hearing students, .41 versus $.47, t(38)=1.02$, $\mathrm{ns}$, while they were marginally different for the deaf students, .30 versus .38 , $t(38)=1.79, p<.10$, according to two-tailed tests. ${ }^{2}$

In short, beyond the greater heterogeneity in their concept knowledge, the deaf students revealed an asymmetry in their category-exemplar relations (stronger links between category members and their categories than the reverse) not evident in hearing peers. Deaf students and hearing students were comparable in the strength of their associative links from exemplars to categories; it was in the links from category names to exemplars that the deaf students showed weaker connections than the hearing students.

\section{Organization and Literacy} Scores of Deaf Students

Correlations between students' reading scores and the frequency with which their responses matched the hearing norms indicated that higher reading scores on the California Reading Comprehension Test, $r(100)=.21$, and the NTID Writing Test, $r(101)=$ .20 , coincided with a higher number of primary associates consistent with the hearing norms (NTID Reading Test, $r(88)=.21, p=.05)$. Correlations between reading scores and the number of words circled as unfamiliar indicated that for the Michigan, California, NTID Reading, and NTID Writing tests, the higher a student's score, the greater his or her vocabulary knowledge, $r(56)=-.68, r(56)=-.57$, $r(52)=-.70, r(56)=-.34$, respectively. The frequency with which exemplars were generated to category stimuli also was inversely related to the number of words circled as unfamiliar, $r(63)=$ -.25 . These weak correlations suggest that either categorical relations are not as vital to literacy as has been suggested (e.g., Cronin et al., 1986) or that college entrance examinations are not the best means of evaluating associative knowledge. In the absence of other information, we tend toward the latter interpretation.

\section{Summary}

Experiment 1 provided additional evidence of considerable overlap in the associative knowledge of deaf and hearing college students. The experiment also revealed an asymmetry in exemplar-category relations among deaf (but not hearing) students and

Table 2

Students' Performance, Analogies Test, Experiment 2

\begin{tabular}{|c|c|c|c|c|c|c|c|}
\hline \multirow[b]{2}{*}{ Analogy } & \multicolumn{2}{|c|}{ Deaf } & \multicolumn{2}{|c|}{ Hearing } & \multicolumn{2}{|c|}{ Combined } & \multirow{2}{*}{$\begin{array}{l}\mathbf{t}(37) \\
p<.01\end{array}$} \\
\hline & $M$ & $S D$ & $M$ & $S D$ & $M$ & $S D$ & \\
\hline Superordinate & 5.11 & 1.88 & 7.90 & .30 & 6.62 & 1.90 & 6.74 \\
\hline Subordinate & 6.33 & 1.33 & 7.52 & .51 & 6.97 & 1.14 & 3.80 \\
\hline Coordinate & 4.06 & 2.15 & 7.38 & .80 & 5.85 & 2.29 & 6.57 \\
\hline Completion/qualifier & 5.00 & 2.33 & 7.00 & 1.10 & 6.08 & 2.02 & 3.52 \\
\hline Rhyme & 3.06 & 2.86 & 7.62 & .74 & 5.51 & 3.04 & 7.06 \\
\hline Part-whole & 4.61 & 1.88 & 6.95 & .74 & 5.87 & 1.81 & 5.25 \\
\hline
\end{tabular}

\section{Experiment 2: A Verbal Analogy Task}

Verbal analogies typically are one of the most difficult parts of standardized, literacy-related examinations for deaf students. One aspect of that challenge is these students' frequently lim- 
ited English vocabulary, relative to hearing peers, a difference that becomes larger rather than smaller through the school years (Traxler, 2000). Also potentially involved are differences in the ways deaf and hearing individuals approach problemsolving tasks and the structure of the analogies used on standardized tests. In particular, research has demonstrated that deaf students generally make less use of relational information than hearing peers in reading, memory, and a variety of other laboratory and real-world tasks (see Marschark, 2003, for a review). It thus seems unlikely that category-exemplar relations are the only domain in which deaf and hearing students' associative knowledge will differ.

Even leaving aside some of the more complex, if not abstruse, kinds of analogies seen on examinations such as the Miller Analogies Test, there may be some kind of relations that are more difficult for deaf than hearing individuals. Based on the findings of Marschark and Everhart (1999), for example, one would expect that analogies in which participants, having been given exemplars, have to provide category names, would fall into that class (e.g., banana : fruit :: rose :__ ). The results of Experiment 1, in contrast, would suggest that analogies requiring exemplar responses to category names would be more difficult for deaf students. Finally, earlier demonstrations that semantic clustering improved the performance of hearing students but not that of deaf students in memory tasks (e.g., Koh et al., 1971; Liben, 1979) suggest that coordinate analogies also might be more difficult for deaf students.

In addition to examining the effects of taxonomic knowledge on the solving of analogies in Experiment 2, we used a normative to ask whether other kinds of verbal analogy (i.e., conceptual relations) might present particular difficulties for deaf students. Experiment 2 was also intended to provide evidence on whether the findings of Experiment 1 and those of McEvoy et al. (1999) and Marschark and Everhart (1999) are of any practical or academic significance. Given the sensitive nature of demonstrating differences between deaf and hearing individuals that have little bearing on educational or vocational performance, this issue is not trivial.

\section{Method}

\section{Normative Study}

The particular kinds of analogies to be examined were determined through a reanalysis of the more than 21,000 single-word associative responses obtained by McEvoy et al. (1999) from deaf and hearing college students. The original data were collected from 136 deaf students from the various colleges of RIT, including NTID, and 136 hearing students from the University of South Florida, via the USF Word Association Norms (Nelson et al., 1998).

The stimuli were 79 common English words selected from the USF norms. All of the responses obtained by McEvoy et al. (1999) initially were compiled into frequency tables. Two of the authors of the present article then independently scored all responses according to association types of the sort described by Deese (1966). One of the scorers was unfamiliar with the research area and intentionally blind to specific predictions, while the other had extensive experience with the Deese methodologies. Most disagreements were resolved in discussion. In the dozen cases (less than $0.06 \%$ ) in which disagreements remained, they were resolved by an English professor at NTID. All responses were then rescored by the two original scorers to ensure consistency with the resolutions of disagreements; $100 \%$ agreement was obtained.

Seven frequently used response sets emerged from the frequency analysis:

- superordinate: canary - bird

- subordinate: airplane - 747

- coordinate: airplane - helicopter

- rhyme: bomb - Tom

- predication: airplane - fast

- part-whole/whole-part: airplane - wing

- sound-related: bomb - tick-tick

An analysis of variance on the frequencies of the various response types indicated a reliable main effect of response set, $F(1,156)=138.49$, but only a marginal effect of hearing status, $F(1,156)$ $=2.83, p<.10$, and no interaction. Correlations between the frequency of responses classified in each response set for deaf and hearing individuals were high and reliable, $r s(21-$ 62) $=.80-.94 .{ }^{3}$ This pattern of results is consistent with the findings of Tweney et al. (1975) and McEvoy et al. (1999) indicating more similarity than difference in the organization of semantic information in the memory of deaf and hearing individuals.

The first six kinds of associative relations in the bulleted list were chosen for use in constructing analogies for Experiment 2. Sound-related analogies were not created because of the possible bias against deaf students in expecting them to know the sounds that things make. Rhyme judgments, in contrast, have been shown to be an appropriate task for deaf college students (Hanson, Goodell, \& Perfetti, 
1991); consequently, they were used in Experiment 2, though rhymes were only infrequently elicited in the word association task.

\section{Participants}

Eighteen deaf and 21 hearing students at RIT participated in Experiment 2. All volunteered in response to flyers or personal contacts and were paid for their participation. Responses from three other deaf students were not scored because these students skipped an entire page of the test booklet.

\section{Materials}

A set of 48 verbal analogies were constructed using category/superordinate, exemplar/subordinate, coordinate, rhyme, part-whole/whole-part, and predication relations (see note 3 ). Eight analogies were constructed for each relation type. The multiple-choice format was identical to that used on standardized tests ( $\mathrm{A}: \mathrm{B}: \mathrm{:} \mathrm{C}:$ __ $)$, with four alternatives for each response. Response alternatives were chosen to ensure that there was only a single correct answer, but that most of the foils were related to one of the terms in the analogy. For predicate and part-whole analogies, half of the items were constructed in the reverse order. The vocabulary used in the analogies was selected with the help of the same experts we called on for Experiment 1.

Six analogies appeared on each page of a eight-page test booklet, one analogy of each type appearing on each page. Otherwise, the analogies were randomly ordered on each page and across pages, and the pages were combined into test booklets in a counterbalanced fashion. Students were tested in groups of varying size. After reading the instructions, which included two practice items, each group was given 10 minutes to complete the booklet. Explanation of the task, re- sponses to questions, and task administration were spoken to hearing students and both signed and spoken to deaf students.

\section{Results and Discussion}

Four hearing students and one deaf student skipped one or more analogies, but there was no apparent consistency or intention in these omissions. Mean correct performance of the deaf and hearing students on each type of analogy is presented in Table 2, where it can be seen that the hearing students obtained significantly higher scores than their deaf peers on each of the six types of analogies. The main effect of hearing status was reliable, $F(1,37)=45.87$, as was the effect of analogy type, $F(1,37)=41.79$, and the hearing status by analogy type interaction, $F(1,37)=11.68$. Overall, pairwise comparisons (using Tukey's honestly significant difference) among the analogy types were all reliable except that performance on predication analogies did not differ from that on coordinate or part-whole analogies; nor did performance on coordinate analogies differ from that on rhyme analogies. The reliable difference of hearing status in solving analogies was particularly interesting given that the deaf and hearing students were relatively similar in their frequency of providing responses of various kinds in the normative task.

The biggest difference in performance between the two groups was in the rhyme analogies, followed by coordinate, superordinate, part-whole, completion/qualifier (predication), and subordinate analogies (see Table 2). Although deaf individuals have been shown to be sensitive to phonological (and orthographic-independent) rhymes, in retrospect, rhymebased analogies are not really an indicator of students' concept knowl- edge, even if they may reflect reasoning skills. The finding that coordinate analogies were particularly difficult for deaf students, however, is consistent with previous studies' findings showing them to be less likely than hearing students to demonstrate taxonomic clustering or benefits from it in free recall (Koh et al., 1971; Liben, 1979). More interestingly, hearing students solved significantly more analogies in which they had to provide superordinate terms (mouse : animal :: poison ivy : plant) than ones in which they had to provide subordinate terms (book : dictionary :: tool : screwdriver), $t(20)=3.51$, whereas deaf students solved significantly more subordinate than superordinate analogies, $t(17)=$ 3.96. This result suggests that knowledge of category superordinates was not as readily applied by deaf students as it was by hearing students (Marschark \& Everhart, 1999). That finding may reflect the lesser stability and coherence in the category knowledge of the deaf students (McEvoy et al., 1999), or it may reflect a greater difficulty in using category knowledge strategically when it is embedded in less familiar contexts (Antonietti, 2001; Goswami, 1992). Consistent with both of these suggestions, Liben (1979) found that deaf children showed just as much semantic clustering in free recall as hearing children, but that deaf children's subsequent recall of those items was less. Liben concluded that although deaf children can recognize and attempt to use the categorical nature of a list to improve memory, they might lack either flexibility in task-appropriate classification of individual items or sufficient knowledge of category membership and structure.

Although we tried to ensure that all words would be familiar to the deaf participants, it seemed likely that the 
Table 3

Correlations of Deaf Students' Literacy Scores and Analogy Performance, Experiment 2

\begin{tabular}{|c|c|c|c|c|c|c|}
\hline & Superordinate & Subordinate & Predicate & Coordinate & Rhyme & Part-whole \\
\hline SAT-V $(n=5)$ & .53 & .77 & .78 & .54 & .76 & $.88^{*}$ \\
\hline ACT English $(n=9)$ & .62 & .62 & $.80^{*}$ & $.67^{*}$ & $.71^{*}$ & $.80^{*}$ \\
\hline ACT Reading $(n=9)$ & .56 & .51 & $.71^{*}$ & .66 & .67 & $.80^{*}$ \\
\hline Michigan $(n=11)$ & $.84^{* *}$ & .73 & $.92^{* *}$ & .60 & $.75^{\star \star}$ & $.82^{* *}$ \\
\hline NTID Reading $(n=13)$ & $.56^{*}$ & .54 & $.75^{* *}$ & .46 & .49 & $.75^{\star *}$ \\
\hline NTID Writing $(n=13)$ & .08 & .35 & .16 & .05 & -.04 & .05 \\
\hline
\end{tabular}

Notes. ACT, American College Test. SAT-V, Scholastic Aptitude Test-Verbal. Michigan, Michigan Test of

English Language Proficiency. NTID, National Technical Institute for the Deaf. California, California

Reading Comprehension Test.

*Correlation significant at the .05 level (two-tailed).

${ }^{* *}$ Correlation significant at the .01 level (two-tailed).

solving of analogies would be related to deaf students' reading abilities. As in Experiment 1, literacy-related admissions and placement scores were available for the deaf students on the ACT, SAT-V, Michigan Test of English Language Proficiency, California Reading Comprehension Test, and NTID placement tests in reading and writing. Scores on these measures were related to performance on the different analogy types, as shown in Table 3. The Michigan and NTID tests were found to be most strongly related to analogy performance, overall, and predication analogies were predicted by the greatest number of tests. Other than the general finding that performance on the analogy test is relatively strongly related to English-language skills, however, there was no pattern of particular interest in the correlations.

The results of Experiment 2 thus indicate that the relative lack of conceptual coherence and heterogeneity in associative knowledge demonstrated by deaf students in several studies (e.g., McEvoy et al., 1999) influences performance on at least one educationally relevant task. Deaf students made significantly more errors in solving analogies than hearing peers, even when given sufficient time to complete the entire test. The particular difficulty of coordinate analogies for deaf students and their solution of more subordinate than superordinate analogies was consistent with results of previous studies indicating that deaf students are less likely than hearing peers to use category information in semantic memory tasks. This result thus demonstrates again the difference between having knowledge and knowing when and how to apply it.

\section{General Discussion}

The two experiments reported in the present article examined the structure and application of taxonomic knowledge. Experiment 1 focused on superordinate-subordinate (categoryexemplar) relations. A single-word association task involved target category and exemplar terms to which hearing students responded most frequently with exemplars and category names, respectively. Responses given by deaf and hearing students revealed an asymmetry in the strength of associations between category terms and exemplars: Deaf students were less likely than hearing students to respond with exemplars when given category names (reptile-snake) than they were to respond with category names when given exemplars (banana-fruit). Such an asymmetry indicates that when encountering a general term, deaf students are less likely than hearing stu- dents to automatically (and likely unconsciously) activate or prime examples of that concept. That difference would affect both language comprehension and any formal or informal learning experience that requires recognition of similarities (or differences) between one concept, object, situation, or event and another.

The application of taxonomic knowledge was explored in Experiment 2 using an analogies task. Despite control of the vocabulary level, deaf students solved significantly fewer of each kind of analogy than their hearing peers. Deaf students correctly solved more analogies in which they had to provide a subordinate term (category member) than analogies in which they had to provide a superordinate term (category label), whereas hearing students showed the reverse pattern.

The results of Experiments 1 and 2 thus were consistent for hearing students; deaf students showed opposite results in the two tasks. At one level, such results reflect the fact that providing responses in a single-word association task is very different from applying category knowledge in a problem-solving task. Analogies not only require activation of word knowledge, they entail identification of possible relations between pairs of 
words (the $\mathrm{A}$ and $\mathrm{B}$ terms of the analogy) and mapping to possible metarelations created by the relation between a third term (C) and multiple-choice (D) alternatives. This transfer of a meaning "package" clearly requires a level of knowledge and analytic skill that goes beyond simple associative connections between individual concepts. Having the conceptual and relational knowledge inherent in any particular analogy does not guarantee that the two forms of knowledge will be successfully combined to yield a correct solution. Further, the processes underlying such performance do not appear to be natural and automatic, but have to be explicitly taught (Antonietti, 2001). It is only through repeated activation and use of those relations that they come to be activated automatically and become part of the strategic arsenal an individual possesses for more complex learning and performance (Bebko \& McKinnon, 1998).

At another level, whereas deaf college students are just as likely as hearing peers to give category names as primary associates to category exemplars, they apparently do not spontaneously apply category structure as often in situations in which doing so would be useful, for example, in solving analogies (Antonietti, 2001). At the level of word/concept knowledge, Goswami (1992, p. 98) noted that when knowledge is "fragmentary," problem solvers will have difficulty resolving the relational information on which analogies are based (Marschark, 2003). The results of Experiment 1 and McEvoy et al. (1999) indicate that deaf students' conceptual knowledge generally is less coherent and consistent than hearing students'. Further, information processing at several levels (including memory for both printed text and sign language) among deaf adults and chil- dren tends to be focused on individual items rather than relations across items (Banks, Gray, \& Fyfe, 1990; Ottem, 1980; Todman \& Seedhouse, 1994). The finding that deaf students' performance on the analogy task used in Experiment 2 was significantly worse than that of hearing students therefore likely reflects differences in both knowledge of category membership and structure and task-appropriate use of relational processing (Liben, 1979).

From both theoretical and applied perspectives, the results of the present study contribute to a better understanding of concept knowledge among deaf individuals. Consistent with a variety of contemporary studies, these experiments suggest that, in some ways, deaf and hearing students' knowledge is similar, at least as reflected in word association tasks (e.g., the normative task of Experiment 2). Still, there are qualitative differences in both organization and application of that knowledge that influence performance (e.g., solving analogies in Experiment 2).

Further research concerning the interaction of language and cognitive performance among deaf students therefore should be instructive both in terms of understanding psycholinguistic functioning and in developing instructional methods appropriate to these students' strengths and needs. Of particular utility would be teaching strategies that encourage deaf students to make use of their knowledge across settings as well as across similar problems (Antonietti, 2001; Goswami, 1992). Among educators of deaf students, it is common to hear about students, for example, who do well in courses such as calculus and trigonometry but fail to apply that knowledge in other courses such as engineering, or who demonstrate knowledge of discourse structure in their signing and writing but fail to use it in reading. While the "transfer of knowledge" problem is commonly encountered in educational settings, it is little understood. As a result, it remains unclear how much similarity in knowledge is "enough" to enable deaf students to perform as well as hearing students, or the extent to which apparently small differences in knowledge or task-related strategies will influence performance on higher-order tasks (Bebko \& McKinnon, 1998).

Such research also should be informative with regard to deaf students' reading challenges. "Knowing" a word means that, after it is processed visually, the reader must retrieve its meaning from memory, including preexisting associative structures (Fischler, 1985; Nelson et al., 1998). These structures depend on past experience, and conceptual understanding of printed text demands this activity. Variability in reading skills and experience, meanwhile, affects the accrual of the mental lexicon, which, in turn, strongly influences reading and learning at large. Analogies, in particular, require a fluid understanding of word meanings and the ability to see various relations among concepts at various levels of hierarchical organization (Liben, 1979), and thus are useful tools for tapping underlying conceptual knowledge and verbal reasoning. If the knowledge inherent in the lexicon is important to achievement in reading, solving analogies, and other academic tasks, then an assessment of its structure in deaf students should help improve reading and academic performance through targeted instructional methods. At the very least, such investigations would enhance understanding of the cognitive processes underlying deaf students' academic performance on such tasks. As Detterman and Thompson (1997, p. 1083) noted, "Lack of under- 
standing of the cognitive skills underlying educational interventions is the fundamental problem in the development of special education."

\section{Authors' Note}

Experiment 1 was conducted as part of a master of science degree program by Carol Convertino, at Rochester Institute of Technology. The authors are grateful to Douglas L. Nelson, Gary Long, Stephen Aldersley and John Albertini for their assistance and advice with regard to several aspects of this project. Correspondence should be addressed to Marc Marschark, Department of Research, National Technical Institute for the Deaf, 96 Lomb Memorial Dr., Rochester, NY 14623 USA (e-mail: memrtl@rit.edu).

\section{Notes}

1. In each of three younger age groups, hearing students scored about $30 \%$ better than their deaf peers. Although the lack of a difference between the deaf and hearing college students could indicate a developmental lag that resolves by early adulthood, it appeared to be more the result of a ceiling effect.

2. Analyses of individual patterns of responding indicated that the frequency with which deaf students' responses to exemplars matched those of hearing students' was significantly related to the frequency with which deaf students matched their responses to category names, $r(113)=.45$. Thus, although there is significant overlap in associative responding in the two directions, the tasks are clearly discrete operations.

3. Subsequent examination of the predication responses with the same independent judge who had resolved scoring disagreements indicated a subclass of responses: those that comprised compound words. Such responses are distinguishable from other predication responses by the creation of an instance that is fundamentally different from others in the category. Thus, street-car and flag-pole are considered compounds because neither fits the description typical of cars and poles, respectively. Street-sign and tar-pit are not compounds, and the target words are simply specifying adjectives. This set was most obvious because differences in the use of compound words suggest differences in vocabulary knowledge, not a surprising finding between deaf and hearing individuals. When the entire predication set was rescored by the original two scorers into compounds and predicates, hearing students were significantly more likely to give compound-word responses than deaf students, $t(156)=$ 2.10 , but the groups did not differ in their frequency of giving other predicate responses, $t(156)=.86$.

\section{References}

Antonietti, A. (2001). Analogical discoveries. Rome: Carroci.

Banks, J., Gray, C., \& Fyfe, R. (1990). The written recall of printed stories by severely deaf children. British Journal of Educational Psychology, 60, 192-206.

Bebko, J. M., \& McKinnon, E. E. (1998). Assessing pragmatic language skills in deaf children: The Language Proficiency Profile. In M. Marschark \& M. D. Clark (Eds.), Psychological perspectives on deafness (Vol. 2, pp. 243-263). Mahwah, NJ: Erlbaum.

Bousfield, W. A. (1953). The occurrence of clustering in the recall of randomly arranged associates. Journal of General Psychology, 49, 229-240.

Chaffin, R. (1997). Associations to unfamiliar words: Learning the meanings of new words. Memory and Cognition, 25, 203-226.

Cronin, V., Pratt, M., Abraham, J., Howell, D., Bishop, S., \& Manning, A. (1986). Word association and the acquisition of reading. Journal of Psycholinguistic Research, 15, 1-11.

Deese, J. (1966). The structure of associations in language and thought. Baltimore: Johns Hopkins University Press.

Detterman, D. K., \& Thompson, L. A. (1997). What is so special about special education? American Psychologist, 52, 1082-1090.

Drinkwater, B. A. (1972). Word associations of aboriginal adolescents: A comparison with Australian and American norms. Australian Psychologist, 7, 190-193.
Emmorey, K. (2002). Language, cognition, and the brain. Mahwah, NJ: Erlbaum.

Fischler, I. (1985). Word recognition, use of context, and reading skill among deaf college students. Reading Research, 20, 203 218.

Goswami, U. (1992). Analogical reasoning in children. Hillsdale, NJ: Erlbaum.

Hanson, V. L., Goodell, E. W., \& Perfetti, C. A. (1991). Tongue-twister effects in the silent reading of hearing and deaf college students. Journal of Memory and Language, 30, 319-330.

Kerr, N. H., \& Johnson, T. H. (1991). Word norms for blind and sighted subjects: Familiarity, concreteness, meaningfulness, imageability, imagery modality, and word associations. Behavior Research Methods, Instruments, and Computers, 23, 461-485.

Koh, S. D., Vernon, M., \& Bailey, W. (1971). Freerecall learning of word lists by prelingual deaf subjects. Journal of Verbal Learning and Verbal Behavior, 10, 542-547.

Landauer, T. K., \& Dumais, S. T. (1997). A solution to Plato's problem: The latent semantic analysis theory of acquisition, induction, and representation of knowledge. Psychological Review, 104, 211-240.

Liben, L. S. (1979). Free recall by deaf and hearing children: Semantic clustering and recall in trained and untrained groups. Journal of Experimental Child Psychology, 27, 105-119.

Marschark, M. (1997). Raising and educating a deaf child. New York: Oxford University Press.

Marschark, M. (2003). Cognitive functioning in deaf adults and children. In M. Marschark \& P. E. Spencer (Eds.), Oxford handbook of deaf studies, language, and education (pp. 464-477). New York: Oxford University Press.

Marschark, M., \& Everhart, V. S. (1999). Problem solving by deaf and hearing children: Twenty questions. Deafness and Education International, 1, 63-79.

Marschark, M., \& Lukomski, J. (2001). Understanding language and learning in deaf children. In M. D. Clark, M. Marschark, \& M. Karchmer (Eds.), Cognition, context, and deafness (pp. 71-86). Washington, DC: Gallaudet University Press.

McEvoy, C., Marschark, M., \& Nelson, D. L. (1999). Comparing the mental lexicons of deaf and hearing individuals. Journal of Educational Psychology, 91, 1-9.

Mitchell, R. E., \& Karchmer, M. (2004). Chasing the mythical 10 percent: Parental hearing status of deaf and hard of hearing students in the United States. Sign Language Studies, 4(2), 138-63.

Moores, D. F. (2001). Educating the deaf: Psychology, principles, and practices. Boston: Houghton Mifflin.

Nelson, D. L., McEvoy, C. L., \& Schreiber, T. A. (1998). The University of South Florida word association, rbyme, and word fragment norms. Retrieved February 3, 2004, from the University of South Florida Web site: http://w3.usf.edu/ FreeAssociation/

Nelson, K. (1999). Levels and modes of representation: Issues for the theory of conceptual change and development. In E. K. Scholnick, K. Nelson, P. H. Miller, \& S. A. Gelman (Eds.), Conceptual development: Piaget's legacy: Vol. 26. The Jean Piaget symposium series (pp. 269-291). Mahwah, NJ: Erlbaum.

Ottem, E. (1980). An analysis of cognitive studies with deaf subjects. American Annals of the Deaf, 125, 564-575.

Strassman, B. (1997). Metacognition and reading in children who are deaf: A review 
of the research. Journal of Deaf Studies and Deaf Education, 2, 140-149.

Strassman, B. K., Kretschmer, R. E., \& Bilsky, L. H. (1987). The instantiation of general terms by deaf adolescents/adults. Journal of Communication Disorders, 20, 1-13.

Todman, J., \& Seedhouse, E. (1994). Visualaction code processing by deaf and hearing children. Language and Cognitive Processes, 9, 129-141.

Traxler, C. B. (2000). Measuring up to performance standards in reading and mathematics: Achievement of selected deaf and hardof-hearing students in the national norming of the ninth edition Stanford Achievement Test. Journal of Deaf Studies and Deaf Education, 5, 337-348.

Tweney, R. D., Hoemann, H. W., \& Andrews, C. E. (1975). Semantic organization in deaf and hearing subjects. Journal of Psycholinguistic Research, 4, 61-73

Vikis-Freibergs, V., \& Freibergs, I. (1976). Free association norms in French and English: Interlinguistic and intralinguistic comparisons. Canadian Journal of Psychology, 30, 123133.

Yoon, C., Feinberg, F., Hu, P., Gutchess, A. H., Hedden, T., Chen, H. Y., Jing, Q., Yao, C., \& Park, D. C. (2002). Category norms as a function of culture and age: Comparisons of item responses to 105 categories by American and Chinese adults. Unpublished manuscript, University of Michigan, Ann Arbor. 